\title{
La mer, lieu de représentation de la femme chez Georges Rodenbach et Théodore Hannon
}

\author{
Estrella DE LA TORRE \\ Universidad de Cádiz \\ estrella.delatorre@uca.es
}

Recibido: $11 / 10 / 2012$

Aceptado: 11/01/2013

Résumé

Rodenbach et Hannon, qui avaient fréquenté la côte belge depuis leur enfance, arrivent à résumer leurs souvenirs dans des recueils poétiques d'une grande beauté. Nous ne pouvons pas affirmer que leurs goûts artistiques et littéraires soient absolument comparables, mais ils manifestent le même attachement à la figure féminine. Idéalisée chez Rodenbach, avec une propension à une certaine misogynie chez Hannon, tous les deux s'obstinent à l'identifier, à l'aide de différentes figures poétiques, avec le paysage maritime, berceau de leurs amours de jeunesse ou de leurs premières expériences sexuelles.

Mots clés: Mer du Nord, femme, poésie, Georges Rodenbach, Théodore Hannon.

\section{El mar, lugar de representación de la mujer en la obra de Georges Rodenbach et Théodore Hannon}

\begin{abstract}
Resumen
Rodenbach y Hannon, que habían frecuentado la costa belga desde su niñez, consiguen resumir sus recuerdos juveniles en unos poemas de gran belleza. No se puede afirmar que haya habido paralelismo en los gustos artísticos y literarios de uno y otro, pero ambos manifiestan la misma atracción por la figura femenina. Idealizada por Rodenbach, propensión a una cierta misoginia por parte de Hannon, ambos se obstinan en identificarla, a través de diferentes figuras poéticas, con el entorno marítimo que vio nacer sus amores juveniles o sus primeras experiencias sexuales.
\end{abstract}

Palabras clave: Mar del Norte, mujer, poesía, Georges Rodenbach, Théodore Hannon.

\section{The sea, a site for the representation of women in the work of Georges Ro- denbach and Théodore Hannon}

\begin{abstract}
Rodenbach and Hannon, who used to visit the coast of Belgium since their childhood, are the authors of poems of great beauty encapsulating their juvenile memories. While it cannot be asserted that their artistic and literary tastes run parallel, both are powerfully drawn to the female figure. Rodenbach tends towards idealization, and Hannon towards a certain misogyny; but both authors repeatedly identify her, through various poetic devices, with the maritime environment that witnessed their budding love affairs and sexual awakening.
\end{abstract}

Keywords: North Sea, woman, poetry, Georges Rodenbach, Théodore Hannon. 


\section{Referencia normalizada}

Torre, E. de la (2013). "La mer, lieu de représentation de la femme chez Georges Rodenbach et Théodore Hannon”. Thélème, Vol. 28, 277-291.

Comme l'affirme Marie Blain-Pinel, la mer constitue pour les poètes un ensemble présymbolique «qu'il revient à chacun d'investir pour lui donner sens, soit en se laissant guider par les stéréotypes d'usage, soit en élaborant une réflexion personnelle au fil d'un mouvement de redécouverte de l'élément » (Blain-Pinel, $2003: 12$ ).

La référence à la mer s'approvisionne par un rapport direct à l'imaginaire des artistes qui leur permet d'exploiter librement le potentiel suggestif qu'elle et tout ce qui l'accompagne et l'entoure (la profondeur, la couleur, ses changements d'état..., les plages) leur inspirent. Comme l'argumente le professeur et linguiste Michel Le Guern, la mer se rattache également à «l'ensemble des données qui constituent l'expérience commune de l'humanité, expérience quotidienne de chacun » (Le Guern, 1973 : 459). Georges Rodenbach, avec La Mer élégante et Théodore Hannon, avec Au clair de la dune, nous ont transmis la même interprétation de la mer $\mathrm{du}$ Nord à travers leurs poèmes. Elle ne sera pas pour eux seulement le point d'accueil des gens de la bonne société fin de siècle pendant la saison estivale, mais aussi et surtout le berceau de leurs amours, l'endroit où la femme se métamorphose et entre en symbiose avec cette mer changeante qui, comme elle, sera capable d'attraper ses amoureux pour leur donner la mort après les avoir possédés. La femme comme la mer sont l'objet de leurs regards, mais celle-ci sera un décor où la femme deviendra le sujet privilégié de leur inspiration.

Rodenbach et Hannon avaient été attirés par la mer du Nord dès leur jeunesse. Comme la plupart des familles bourgeoises belges, ils allaient passer leurs vacances d'été à Ostende et, entourés d'une société « élégante », ils y trouvaient l'inspiration pour leurs travaux artistiques. Il n'y avait que quatre ans de différence entre eux, mais une différence abyssale séparait leur caractère et leur manière de percevoir ces périodes de vacances.

Pour Hannon, Ostende et ses habitants saisonniers des années quatre-vingt étaient une source d'inspiration pour composer ses tableaux de jeunesse, comme Iwan Gilkin nous l'évoque dans ses Mémoires inachevés :

\footnotetext{
Quand il m'invitait à l'accompagner dans ses expéditions à la recherche d'un paysage à peindre, je me gardais bien de refuser. Théo, la boite au dos, parcourait à grandes enjambées la digue ou la plage, escaladait les dunes ; toujours à la recherche de son point de vue, s'arrêtant un moment, se faisant de la main gauche une visière, de la droite découpant dans l'espace des carrés imaginaires qui représentaient d'avance le cadre du futur tableau (Gilkin, $2000: 270$ ).
}

Pour Rodenbach, Ostende et ses plages lui donnèrent l'occasion de fonder une petite revue. Accompagné de son ami Emile Verhaeren, ils décidèrent de créer un journal pour divertir la population croissante des touristes. C'est ainsi que pendant les deux étés de 1882 et 1883, tous deux, alors jeunes avocats, qui s'étaient déjà 
initiés au monde de la littérature, remplirent presqu'à eux seuls les colonnes de la revue La Plage. La majorité des poètes de La Jeune Belgique qui devaient soutenir ce projet y prirent une part occasionnelle, à commencer par Max Waller. L'Art Moderne ne manqua pas l'occasion pour lui consacrer sa «Petite Chronique » du numéro 30 du 3 juillet 1882, ajoutant une succincte critique à La Mer élégante de Rodenbach :

Un nouveau journal vient de paraitre. Titre : La Plage, journal mondain. Ses parrains sont MM. Georges Rodenbach et Émile Verhaeren, deux jeunes littérateurs de mérite, qui se chargeront de donner au nouveau-né une bonne éducation et de le présenter avantageusement dans le monde. On connait, du premier, les Tristesses et la Mer élégante, un joli recueil de vers dans lequel l'auteur a mis quelque chose de son amour pour les grands horizons, la ligne austère des dunes, les séductions de la jetée. Nul doute [sic]que La Plage, avec cette collaboration, ne soit intéressante et bien écrite. Trois numéros ont paru. Hebdomadaire, le journal est destiné à devenir prochainement quotidien. Nous lui souhaitons vie et succès (L'Art Moderne, 1882, n 30. p. 239).

On y retrouvera sa signature et celle d'Iwan Gilkin au bas de nouvelles légères, divers billets aux noms d'Henry Maubel, de Théo Hannon et d'Albert Giraud, ainsi que des poèmes composés par le peintre Georges Khnopff. Faute d'une équipe permanente, Rodenbach et Verhaeren se servaient d'une panoplie de pseudonymes quand ils n'inventaient pas des correspondants inexistants.

La Plage comptait parmi ses collaborateurs plusieurs écrivains qui avaient déjà participé à $L a M e r$, une publication du même genre, lancée l'année précédente. La filiation entre les deux revues apparaît dès l'article inaugural du 2 juillet 1882 dans lequel Rodenbach définit clairement le ton et le contenu des douze pages du nouveau journal. Outre la publicité et la liste des étrangers en villégiature à Ostende, Blankenberghe, Heyst, La Zoute ou La Panne, les vacanciers pouvaient s'aventurer en toute confiance dans ces colonnes et parcourir les différents billets les informant de tous les spectacles jusqu'au bal le plus discret, de chacune des courses de l'été, et surtout des commérages qui n'arrêtaient pas de circuler dans cette petite élite de bourgeois.

Un an avant l'apparition de La Plage, Alphonse Lemerre, éditeur parisien, publiait un recueil de trente-cinq poèmes de Georges Rodenbach, La mer élégante, il n'avait que vingt-cinq ans; Théodore Hannon, ancien collaborateur de La Plage, vingt-huit ans plus tard, en 1909, publiait à Paris, chez Dorbon aîné, les quarante poèmes de son recueil Au Clair de la dune.

En réalité, Hannon ne composa pas un recueil où tous les poèmes étaient inédits, il y récupéra quelques-uns qui avaient appartenu à d'autres œuvres. " La Mer enrhumée», « Jaloux » et « Citrons » sortaient de Rimes de joie ; «L'Éventail » de Les Vingt-quatre coups de sonnet, et « Fruits de mer» n'était que la transcription presque exacte de la «Marchande de marée » de Au pays de Manneken-Pis.

Malheureusement pour Théodore Hannon, la reconnaissance internationale de son cadet et l'oubli et le discrédit où il était tombé après ses Rimes de joie et sa rentrée dans le monde de la revue musicale, jouèrent contre lui. Pour la critique moderne $A u$ clair de la dune ne renferme aucun intérêt car il n'est qu'une répétion de ce que Rodenbach avait déjà fait. Pour Christian Berg : " Il se borne à ressasser 
un thème déjà bien traité par Georges Rodenbach dans La mer élégante » (Berg, 1988 : 320) ; et pour Paul Delsemme il constitue un: «joli sujet, mais pas neuf, traité en vers par Georges Rodenbach » (Delsemme, 1999: 193).

Et pourtant, La Mer élégante, considérée comme le modèle, n'a jamais fait partie des chefs-d'œuvre de son auteur. Attaqué par la critique contemporaine, l'auteur lui-même allait la renier. Quand il était en train de rédiger ses vers, Rodenbach adresse une lettre à son ami Potvin, où il banalise son recueil, il n'y voit que la description de " ce côté tout nouveau et tout moderne des Villes de Mer : la vie mondaine avec ses concerts et ses bals, avec ses marivaudages dans les dunes et les villas "; même si plus tard, dans une autre lettre qu'il écrit sur le même sujet à Jules Eyerman (datée du 8 décembre 1880), il s'assigne une tâche plus élevée, il reconsidère ses premiers mots pour y voir quelque chose de plus sérieux:

Je me préoccupe encore de poésie, et je vous annonce même que je publierai peut-être l'été prochain un nouveau volume intitulé La Mer élégante. C'est la peinture de la vie mondaine de concerts et de bals qu'on mène au bord de la mer. Quelque chose comme du Van Beers ou du Stevens. C'est là en poésie un genre nouveau, car jusqu'ici les poètes n'ont vu près des flots que les matelots et les barques de pêche. Dans ce cadre nouveau, j'ai placé l'homme éternel: l'homme qui doute et qui croit, l'homme qui rêve et qui aime. J'ai fait cela d'un trait au retour d'une villégiature à Blankenberghe (Bodson-Thomas, 1942 : 29-30).

Le succès de La Mer élégante fut inférieur à celui de son antérieur recueil Tristesses. Les rares publications qui s'intéressaient à la littérature, comme Le Journal des Gens de Lettres Belges, La Revue de Belgique, Le Journal du dimanche (supplément hebdomadaire du quotidien bruxellois L'Europe) reproduisaient quelques poèmes du livre en les accompagnant d'un commentaire élogieux. Dans Le Journal $d u$ dimanche, Camille Lemonnier qui signait «Un Liseur», fait un vif éloge de l'œuvre de Rodenbach dans le numéro du 24 juillet 1881. Il déclare : «La Mer élégante est une des plus fortes jouissances littéraires qu'il m'a été donné de goûter depuis longtemps ». Il range son auteur parmi « les bons artistes de n'importe quel pays ». Il le trouve même supérieur à François Coppée "par le choix et l'éclat des images, la couleur du sentiment, l'abondance de l'idée, la sûreté et la finesse de la touche... » (Maes, 1952: 82). La critique d'une petite revue namuroise Plume et crayon que signait Auguste (Auguste Mestdagh) ne confirma pas le jugement de Camille Lemonnier, bien au contraire (Maes, 1952: 82-83). Son article plutôt malveillant lui valut deux lettres indignées de Rodenbach, publiées dans Plume et Crayon des 20 août, 5 et 20 septembre 1881. L'Art moderne, dirigée par Edmond Picard, parle « d'observation superficielle et parfois fausse, comparaisons forcées, mots arrivant à la fin des vers par besoin de rimer mais non par raisons " ( $L^{\prime}$ Art Moderne, n'21, 24 juillet 1881, pp: 164-165), mais Max Waller insiste sur l'évocation " couleur de pastel... de la vie ensoleillée des plages à la mode » (Gilsoul, 1936 : 127) qu'il voudrait voir illustrer par Watteau ou par Boucher. Anny Bodson-Thomas considère ce recueil comme un accident dans l'œuvre poétique de Rodenbach, pour conclure que «La Mer élégante est plutôt symptomatique de 
l'incapacité de comprendre la nature chez le poète dont l'attention se dirigera par la suite exclusivement vers les villes » (Bodson-Thomas, 1942:30).

Mais, malgré cet accueil peu édifiant et bariolé, Georges Rodenbach s'était proposé de bâtir une œuvre sur une esthétique bien conçue, que la citation de Balzac qui constitue l'épigraphe de son recueil, confirme: «L'oisif mène la vie élégante ; l'artiste la crée parce qu'il la sent» (Rodenbach, 1881: 2). Trois ans après, l'écrivain se réitère dans ses déclarations à travers le fragment de Goncourt qui ouvre son Hiver mondain recueil dont les propos diffèrent très peu de ceux de $L a$ Mer élégante :

Le Réalisme n'a pas l'unique mission de décrire ce qui est bas, ce qui est répugnant ; il est venu au monde aussi, lui, pour définir dans de l'écriture artiste ce qui est joli, ce qui est élevé, ce qui est bon, et encore pour donner les aspects, et les profils des êtres raffinés et des choses riches (Rodenbach, $1884: 8)$.

Le jeune écrivain s'impose la tâche de recréer, sous une forme réaliste mais aussi intimiste, des décors mondains qui renferment la représentation d'histoires personnelles dont il s'avère être le protagoniste. Sans laisser de côté l'écriture artiste et le réalisme des descriptions, il y déploie le rêve par-dessus le réel.

Rodenbach, qui allait devenir avec le temps l'auteur des villes, d'une ville en particulier, Bruges, mais d'une Bruges réinventée, éloignée de la modernité qui s'imposait à elle malgré elle, une Bruges décrite à partir du regard des hommes envoutés par l'amour, se montre déjà un être double à vingt-six ans, en récupérant les plages belges à la mode. D'une part, il se complait à décrire la réalité de ces plages qui s'ouvraient à une population avide d'amusements, de faire la démonstration de sa supériorité face aux habitants habituels. Mais d'une autre, il cesse d'être frivole pour nous montrer le poète intimiste qui intériorise sa perception de la plage et de la mer en fonction de ses expériences amoureuses. Une femme ou plusieurs, mais un seul sentiment qui s'impose à lui pour transpercer une réalité qui se transforme en fonction de la femme aimée et de la fin heureuse ou malheureuse de l'expérience vécue.

Pour Théodore Hannon la femme n'a été que rarement un sujet idéalisé, pour le poète-peintre elle restait un objet de désir sexuel, un bel objet à regarder et utiliser. À travers les poèmes de Au clair de la dune, le poète se complait à l'utilisation de scènes maritimes ou de décors mondains de la plage d'Ostende pour y poser ses regards lascifs à la recherche de belles femmes surprises dans des moments où se montrent tous leurs charmes physiques.

La charge érotique du recueil Théodore Hannon nous l'annonce déjà dans le titre. Il récupère, en substituant la «lune» par la « dune», celle d'une vieille chanson populaire française anonyme du XVIIIe siècle, attribuée parfois au musicien JeanBaptiste Lully. Apparemment innocents les mots de la version originale renferment des clés qui l'identifient comme une chanson chargée de propos érotiques :

Au clair de la lune,

Mon ami Pierrot,

Prête-moi ta plume 
Pour écrire un mot.

Ma chandelle est morte,

Je n'ai plus de feu ;

Ouvre-moi ta porte,

Pour l'amour de Dieu.

Au clair de la lune,

Pierrot répondit :

«Je n'ai pas de plume,

Je suis dans mon lit.

Va chez la voisine,

Je crois qu'elle y est,

Car dans sa cuisine

On bat le briquet. »

Au clair de la lune,

L'aimable Lubin;

Frappe chez la brune,

Elle répond soudain :

- Qui frappe de la sorte?

Il dit à son tour :

- Ouvrez votre porte,

Pour le Dieu d'Amour.

Au clair de la lune,

On n'y voit qu'un peu.

On chercha la plume,

On chercha le feu.

En cherchant d'la sorte,

Je n'sais c'qu'on trouva ;

Mais je sais qu'la porte

Sur eux se ferma.

Si des termes comme «Lubin » (moine dépravé) et le « dieu d'Amour » induisent des sous-entendus sexuels, des métaphores comme « rallumer le feu » (l'ardeur) lorsque « la chandelle est morte» (le pénis au repos), en allant voir la voisine qui «bat le briquet» (désigne l'acte sexuel), peuvent être interprétées de façon lubrique.

La Mer élégante détaille en réalité le rapport jour à jour de l'évolution d'une belle histoire d'amour dont le protagoniste est le poète. Rodenbach introduit son recueil par un « Prologue » où, à la manière d'un article de journal, il nous décrit les raisons qui poussent les gens de la bonne société à quitter leur ville pour aller vers la mer, une mer au sein de laquelle tout change grâce à ses nouveaux habitants, mais le beau paysage donne libre cours à l'amour, amour qui s'impose dans les cœurs des filles :

Mais voici qu'un beau jour toutes ces jeunes filles Jasant sous l'éventail, sans souci, sans désir,

Sentiront que leur cœur caché sous leurs mantilles A besoin de tendresse autant que de plaisir

(Rodenbach, 1881:5). 
Le jeune poète ne sera au début qu'un spectateur attentif, il se complaira à voir passer les belles femmes qui peuplent les plages, mais, si au début il ne les considère que comme des femmes « mièvres », ses sens s'ouvrent à la réalité du désir :

\footnotetext{
Je voudrais que mon cœur vibrant de poésie

Fût grand comme un sérail pour toutes les aimer

Et qu'il pût à son gré comme un prince d'Asie

Dans un harem d'amour toutes les enfermer
}

(Rodenbach, $1881: 12$ )

Attiré par toutes les jeunes filles de vingt ans qui se promènent le long de la plage et de la digue, il est conscient que son idéal féminin n'est qu'un rêve impossible à réaliser : " Où donc es-tu, mon idéal ?/ Où donc es-tu, femme rêvée ? » (Rodenbach, $1881: 15)$. La rencontre ne tardera pas à se produire. Il approche d'une jeune inconnue : "Charmante enfant aux cheveux bruns » (Rodenbach, 1881:16), avec l'excuse de lui demander si elle aime les fleurs et les sonnets la réponse est immédiate : " "Faites-en un pour moi", dit-elle» (Rodenbach, 1881:16). Dans le sonnet qui suit cette première " Rencontre », le poète nous fait son beau " Portrait », et même s'il la trouve encore " insouciante »: «Elle est insouciante encore comme un enfant» (Rodenbach, 1881: 17), elle l'aime malgré tout, même s'il n'est pas encore sûr d'être correspondu :

Oh ! si la chère enfant pouvait voir mon émoi Et combien en amour les hommes sont vulgaires Elle m'aimerait mieux et n'aimerait que moi

(Rodenbach, 1881 : 17).

Sa belle histoire continue et, dans l' "Aveu », il nous transcrit le grand moment où il lui avoue son amour. En se comparant à deux grands amoureux de la littérature, Roméo et Faust, comme eux, il croit avoir rencontré sa « himère » et son " rêve », comme eux : « un soir [...], devant la mer sans voiles/ [...] / J'ai trouvé l'idéale enfant que je rêvais » (Rodenbach, 1881 : 37). Mais la fin du poème laisse entrevoir un certain pessimisme, sans le vouloir, il prévoit la fin de cet amour qui vient de se montrer à lui, même si en attendant il se voit marié : «Et sans prévoir qu'un jour il pourrait se briser, /J'ai caressé ce rêve où je l'aurais pour femme/ Le front couvert d'un voile aussi blanc que son âme !» (Rodenbach, 1881 : 37). Dans le sonnet qui suit, "L'Oubli », le jeune amoureux est conscient que cet amour, comme tous ceux qui naissent en été au bord des plages, ne va pas perdurer : " $C$ 'est en vain qu'on s'épuise en regrets superflus !/ Ces amours sont pour nous comme ces coquillages / ternis quand l'air marin ne les avive pas !... » (Rodenbach, 1881 :39). Mais le poète se «Révolte contre l'oubli », même s'il s'avoue un amoureux volage, incapable d'admettre que l'amour puisse s'oublier, il s'attache à la possibilité d'amours perdurables : "Que l'amour peut durer et que l'homme peut boire / Toujours au même verre, et des siècles entiers !...» (Rodenbach, 1881: 42). Dans «Lied », le poète consacre une petite chanson à sa conviction que la réalité de l'amour n'existe que dans les rêves, c'est dans ces moments que l'inconscient de l'amoureux atteint le 
bonheur absolu : " Aussi pour la revoir sans trêve / Et pour attiser notre amour, / Puisqu'en dormant toujours j'en rêve, / Je voudrais dormir tout le jour » (Rodenbach, $1881: 65$ ).

Rodenbach profite de la description d'une après-midi passée « Dans les dunes », échappant à la chaleur, pour nous transcrire une expérience vécue avec sa bienaimée. Expérience poétique car toute l'histoire s'appuie sur les vers d'amour d'autres poètes (Brizeux, Dante et Pétrarque) qui, comme lui, furent inspirés par des femmes qu'ils adoraient en les idéalisant. Conscient de son infériorité, il se reconnait un seul parallélisme avec eux, être amoureux:

\footnotetext{
O toi que j'aime tant ! Que ne puis-je à mon tour Te chanter d'un cœur ferme et d'une voix ardente Comme a chanté Pétrarque et comme a chanté Dante, Puisque, sans leur génie, au moins j'ai leur amour !... (Rodenbach, $1881: 69$ ).
}

$\mathrm{Au}$ fur et à mesure que Rodenbach avance dans le recueil et dans la transcription de son histoire sentimentale, son pessimisme face à l'évolution de son amour commence à se faire sentir "Fatigué de ce monde élégant et frivole », un soir, le poète se met devant la mer pour inventer une « Fantaisie céleste », où il imagine d'autres mondes peuplés comme le nôtre, où il se voit changé par le phénomène de la métempsychose en un être bon, doux, indulgent, soumis. Comparant le ciel à un océan peuplé d'étoiles semblables à de blanches voiles, son rêve finit dans l'exaltation de pouvoir récupérer la femme «que j'aimais et qui m'aimait jadis », et vivre éternellement avec elle: "Ce serait tout mon ciel et tout mon paradis!» (Rodenbach, 1881: 108).

Son histoire d'amour finira avec l'été, Rodenbach se sert d'un « Épilogue » pour la clôturer ainsi que le recueil. Ce dernier poème sera affecté par la nostalgie et le spleen. À travers chaque vers le poète mettra en rapport la fin de la saison et celle de leur amour, mais tandis que l'été revient toujours, l'amour « est mort sans qu'il puisse renaître $»$ :

\section{Seul notre amour est mort sans qu'il puisse renaître ; \\ Le roman est perdu, sans qu'il soit achevé ; \\ Pourtant j'avais donné le meilleur de mon être \\ Pour qu'il fût aussi beau que je l'avais rêvé}

(Rodenbach, 1881 : 111).

Le dernier quatrain du poème résume les intentions du jeune poète au moment de concevoir son recueil, définitivement non satisfaites :

\footnotetext{
J'ai tissé chaque vers comme une bandelette

Pour te garder intact et pour t'éterniser ;

Pauvre amour ! Dors en paix dans ta blanche toilette ;

Reçois mes derniers pleurs et mon dernier baiser !

(Rodenbach, 1881 : 112).
} 
La belle esquisse d'une histoire d'amour que Rodenbach dessine dans un ouvrage qui se présentait comme apparemment frivole, se complète par une série de poèmes où le jeune écrivain qui s'initiait au monde littéraire va déjà introduire ce qui allait illustrer son esthétique. Toute sa poésie et sa prose vont se caractériser par le procédé impressionniste appliqué à la littérature, impressionnisme circonscrit dans une atmosphère de spiritualisme et de symbole. Dans la Mer élégante le poète n'a jamais oublié les espaces, les personnages, les objets qui furent témoins de son histoire sentimentale. Les descriptions abondent, mais il s'agit de descriptions faites toujours à travers le regard d'un artiste obsédé par l'amour. Les identifications entre ce sentiment, la femme, les objets et les perceptions sensorielles sont constantes. Les amours perdus et les parfums se rapprochent et se font poème chez Rodenbach. Il les aime pour s' " alanguir les sens et pour rêver/ à des amours douces ou fortes 》. Il arrive à rapprocher les différentes odeurs de chaque étape de l'amour: avant l'amour, l'ambre et la verveine; pour l'extase des baisers, les vagues parfums ; pour l'amour déjà fini, le musc ou la rose. Il avoue son dernier souhait avant de mourir, conférant ainsi aux parfums le pouvoir de l'immortalité : sentir toutes les odeurs capables de plonger son esprit dans les rêves plus que le vin ou la musique :

Près d'agoniser sur ma couche

Qu'on m'offre des flacons d'odeur et qu'à mon grès

L'un après l'autre on les débouche.

Car je pourrai — grisant mon esprit expirant

Par ces senteurs douces ou fortes-

Rêver d'éternité future en respirant

L'âme immortelle des fleurs mortes !...

(Rodenbach, $1881: 29$ ).

Le clavier d'un piano écouté pendant un concert lui suggère le parallélisme entre l'effet produit sur ce clavier quand il est touché par les doigts d'une pianiste et celui produit dans l'âme de l'homme amoureux quand elle est touchée par la bien-aimée :

\footnotetext{
Or ce clavier sonore est pareil à notre âme :

Comme lui blanche et noire, elle a toute la gamme

Des chantantes vertus et des vices grondants ;

Il lui suffit aussi pour qu'elle vibre et pleure

Et donne tout l'amour qu'elle cache au-dedans,

Il suffit que la main d'une femme l'affleure!
}

(Rodenbach, $1881: 34$ ).

Dans «Valses rêvées » Rodenbach, qui n'a jamais quitté les lieux élégants, décors de son amour, se sent inspiré par le bal à la mode, les valses. Après s'être questionné sur les propos cachés dans l'âme de leurs compositeurs au moment de les écrire, il conclut qu'elles furent construites : «Pour qu'en les entendant le soir au bord des flots/ les vierges de seize ans qu'on n'a pas fiancées/ Comprennent tout à coup que leur rêve est éclos » (Rodenbach, 1881 : 44). 
Les quatrains du poème «Les Régates » nous décrivent pas à pas l'ambiance d'un après-midi où des jeunes canotiers se divertissent en amusant les spectateurs et surtout les spectatrices. Scènes réalistes qui arrivent à nous transporter dans les ambiances élégantes des plages, mais d'où l'amour ne pouvait pas rester absent : « Allons ! canotiers! vivent les ivresses ! / [...] / Vous irez ce soir avec vos maîtresses / Vainqueurs et vaincus, -remplir les hôtels » (Rodenbach, 1881: 47). En se souvenant de la fameuse Carte de Tendre, Rodenbach revendique la fin heureuse d'une heureuse journée : "Vous naviguerez gaîment jusqu'au jour / Sur le fleuve Joie au Pays du Tendre /Où vous choisirez pour barreur l'Amour! » (Rodenbach, $1881: 48)$.

« Promenade en mer » renferme un autre parallélisme entre un fait banal et habituel des ports de mer en été, une promenade en bateau, et l'évolution des histoires d'amour. Toutes deux commencent bien, mais un mauvais temps est capable de les tourner en disgrâce. Le poète conclut :

\author{
Je songeais : C'est ainsi du voyage d'amour. \\ Au matin de sa vie on s'embarque un beau jour \\ Les mains pleines de fleurs, et le cœur plein de rêves ; \\ Mais à peine s'est-on élancé loin des grèves [....] \\ que le charme vous quitte et la douleur vous prend \\ (Rodenbach, $1881: 73$ ).
}

L'idéalisation de la femme qui parcourt tout le recueil, pourrait se résumer dans « Les Cocottes » où, Rodenbach, après s'être réjoui à la vue des belles courtisanes qui se promènent le soir le long de la digue, nous montre ses préférences féminines pour les jeunes vierges qu'il métamorphose en « livres nouveaux » :

\footnotetext{
Car ces cocottes-là sont comme des romans

Richement reliés, mais souvent assommants

Qu'on se prête et qu'on lit dans l'ennui des voyages!

Aussi vaut-il mieux prendre au temps du renouveau

Une vierge qui soit comme un livre nouveau

dont on est le premier à découper les pages !...
}

(Rodenbach, $1881: 59$ ).

Ce poème de Rodenbach, confronté à «La gloire des Lâches » des Rimes de joie de Hannon, où le poète proclamait son attraction pour les courtisanes :

\footnotetext{
Vivent ces discrètes servantes

Attentives à nos désirs

En assaisonnant nos plaisirs

De complications savantes !

Jour et nuit pour nous pavoisé,

Leur idéal qui vit de prose

Sans épines nous tend la rose...

C'est l'amour fauve apprivoisé

(Hannon, 1884: 35).
} 
exemplifie la distance qui existe dans leur manière de considérer la femme, et renferme la clé des oppositions nettes entre leur manière de réinterpréter la figure féminine à l'intérieur des espaces maritimes. Rodenbach les refait, les réinvente en fonction de son idéal d'amour, il s'éloigne des petits tableaux naturalistes que Théodore Hannon introduit dans Au clair de la dune.

En parcourant les pages du recueil d'Hannon, nous ne pouvons pas oublier que le poète ne cessa jamais de peindre. Aquarelliste et graveur, l'écriture, reléguée au second plan, pouvait être traitée comme un passe-temps. Théodore Hannon avoua à plusieurs reprises qu'il se voulait peintre avant d'être écrivain. La mer du Nord exerça un profond attrait sur de nombreux peintres de l'école réaliste, Ensor, qui le connaissait bien, évoquera ses fines études du vieil Ostende exécutées vers 1880 :

De Théo Hannon, des vues du vieil Ostende exécutées vers 1880, très fines études où les bleus doux, les gris éteints voisinent spirituellement. Bouquet subtil et délicat, chantant la beauté ingénue du vieil Ostende, aujourd'hui disparu, hélas ! Pauvre vieil Ostende, livré aux déprédations d'architectes boiteux à la vue basse. L'absence de procédés roublards accentue le caractère des œuvres de Hannon et les sympathies inclinent vers lui tout naturellement (Ensor, 1921:40).

Camille Lemonnier, qui lui consacre quelques lignes dans sa Vie d'écrivain, souligne l'importance de son côté peintre en le comparant à Félicien Rops : « Il fut bien en poésie une manière de Rops déluré, moqueur, mousseux et érotique » (Lemonnier, 1945 : 140). La collaboration entre les deux artistes, qui commença avec la première l'édition des Rimes de joie, continua tout au long de leur carrière.

Ostende, considérée par Hannon comme une "ville de luxe", "la Reine des plages », d'où était sortie "Venus la Blonde », lui sert de toile de fond pour y insérer les différentes figures féminines. L' «Eau bénite » de la mer du Nord attire les jeunes «beaux diables» car «L'amour, fuyant les entresols, / Flirte, ô gué ! sous les parasols » (Hannon, $1909: 16$ ).

Dès le poème préliminaire, Hannon fait parler une femme, sa "Muse», une jeune baigneuse « ultra-moderne », dans "son maillot de bain» et " aux hanches les deux poings » pour crier le «Boniment» qui nous présente le recueil fait en l'« honneur du littoral » et de «l'exquis féminin » :

\footnotetext{
L'album qu'ici je vous présente

Est fait de soleil et de vent,

De l'écume phosphorescente

Et des soupirs du flot mouvant...

Si quelque quatrain te la coupe,

Benoît lecteur, sois tolérant,

Car je fis ces vers en tirant

(Ah ! l'exquis féminin) ma coupe

(Hannon, 1909: 6).
}

Le voyeurisme se fait art à travers les poèmes du recueil. Hannon développe amplement l'érotique du regard en attrapant des instantanés où les femmes se 
montrent dans toute leur splendeur sous leurs robes claires et transparentes soulevées par le fort vent du Nord: « Car du Nord le souffle suspect / Trousse et retrousse sans respect... / Et nous nous rinçons les mirettes » (Hannon, 1909 : 59) :

\footnotetext{
Ce vent, grand retrousseur de filles, sur la digue

S'amuse... Son haleine indiscrète en soufflant

Plaque l'étoffe et moule et torse et rable et flanc.

Il moule, et lors devient sculpteur, sculpteur prodigue,

Il moule, et nous pouvons nous payer, éblouis,

Des Tanagra de chair - et vivants - un louis !

(Hannon, 1909: 82).
}

Ce vent du Nord qui lui permet de voir les «Mollets »: «Deux par deux, nerveux ou replets, / Dans les bas à jours, les mollets / Vont cambrant leurs rondeurs jumelles » (Hannon, 1909 : 64).

Guy Rosolato considère l'aspect génétiquement visuel du fétiche ${ }^{1}$. Quand Hannon devient fétichiste le désir sexuel du poète se fixe sur un objet lié aux femmes qui fréquentent les plages d'Ostende. «L'éventail » que les dames portaient au côté "Comme la dague moyen-âge », pendant les soirées de bal, devient un "éventail des fièvres » qui bat sur les lèvres féminines, pour baiser leur bouche. Ce n'est pas seulement le vent du Nord qui devient complice des regards obscènes du poète, mais aussi les « Robes claires » à travers lesquelles «L'œil à l'aise suit les contours » (Hannon, 1909: 53). À partir d'un accessoire féminin, le voile, Hannon associe le mysticisme et le profane et compare la plage à un «couvent» dans le sonnet « Voiles de plage» :

\footnotetext{
La plage est le couvent des cœurs

Où ces dames prennent, en chœurs, Paradoxalement le voile ! (Hannon, 1909: 86).
}

L'identification de la mer avec la femme deviendra une constante. Si dans « Profanes », la mer est une coquette «Dont l'homme n'a jamais su faire la conquête, /

\footnotetext{
${ }^{1}$ Guy Rosolato est sans doute celui qui a le plus insisté sur l'aspect génétiquement visuel du fétiche et sur ses affinités ambivalentes avec l'« objet de perspective », en fonction duquel s'ordonnent, selon lui, tous les mécanismes de substitution propres à la pensée inconsciente. Quand, dans un texte littéraire, une description procure des gratifications visuelles qu'il est possible de mettre en relation avec une structure psychique marquée par un recul devant ce qui, dans la différence des sexes, constitue une menace de castration, il est difficile de ne pas y reconnaitre un fonctionnement de l'image sous le régime du fétichisme. Cf. Rosolato, G., (1967) "Étude des perversions sexuelles à partir du fétichisme" in Rosolato, G. et al. Le Désir et la perversion. Paris, Seuil, pp. 9-40 ; Rosolato, G., (1970) "Le fétichisme dont se dérobe l'objet" in Le Champ visuel. Nouvelle Revue de Psychanalyse. №2, pp. 31-39 ; Rosolato, G., (1987) "L'objet de perspective dans ses assises visuelles" in Le Champ visuel. Nouvelle Revue de Psychanalyse. $\mathrm{N}^{\circ} 35$, pp. 143-164.
} 
Cruelle, elle se rit de lui » (Hannon, 1909: 26), d'autres fois, il s'apprête à la personnifier, il peut l'imaginer enrhumée à la manière d'une femme : « la nuit, elle dort toute nue, $[\ldots]$ et la nue / Crève, glaçant son ventre et ses seins frissonnants » (Hannon, 1909 : 38). Personnification que Hannon étendra à une dune qui : " Aux baisers du soleil, sans craindre sa brûlure, / La dune nue étale en riant ses rondeurs » (Hannon, 1909 : 28).

Fidèle à ses principes baudelairiens, Hannon se complait à produire une fusion entre le paysage et l'aimée, particulièrement à travers le regard féminin, volontiers associé à la mer. Parfois il établit une analogie par une contamination lexicale entre le regard et l'océan. Dans « Mer fâchée » les yeux de la femme sont des gouffres où l'homme se noie, et dans « Pieuvre» : « Ils rappellent, vos yeux, la mer profonde et brune/ La morne mer des nuits sans lune » (Hannon, 1909:25) ou bien, il croit que les yeux noirs de l'aimée sont nés de la noirceur de l'océan :

\section{Dans l'océan, un soir, un dense soir d'orage, \\ Satan a dû puiser le féerique cirage \\ De ces diamants noirs au ténébreux éclair}

(Hannon, 1909 : 44).

Hannon pousse loin ses propos érotiques, son univers imaginaire lui fait concevoir la mer comme une rivale dans sa bataille pour conquérir les femmes. Dans « Heure du bain », il imagine des vagues « lascives» capables d'enlacer les corps blancs des baigneuses. Mais c'est dans un des plus longs poèmes de son recueil, " Jaloux », récupéré de la deuxième édition de ses Rimes de joie, que Théodore Hannon construit un bel exemple de poésie au thème lesbien, à tel point sont illustratives les images métaphoriques qu'il y emploie. Toute la séquence textuelle constitue une mise en place, une mise en scène, une mise en tableau. Dans chaque quatrain il imagine, à travers un regard lascif, la possession que la mer fait peu à peu du corps d'une belle baigneuse. En commençant par les pieds, le « flot » encerclera de «clairs anneaux » ses chevilles. Mais la volupté de la mer amoureuse augmente quand elle atteint les cuisses et commence «l'assaut» de la poitrine, les seins de la baigneuse seront «dardés de leurs mille langues ». Finalement l'acte s'accomplit, la possession est totale :

\footnotetext{
Plus indiscrète qu'un amant, La vague aux lesbiennes ivresses,

T'enveloppait étonnamment

De ses infécondes caresses.

Puis enfin, la mer t'engloutit Enamourée, âpre, béante, Te roulant, pâmée, en son lit D'un baiser de Sapho géante (Hannon, 1909 : 57).
}

L'objet le plus banal peut comporter une valeur érotique aux yeux du poète; les coquillages sont souvent réinterprétés par Hannon, mais c'est dans les quatrains de 
« Coquillages » que le poète inscrit de façon évidente les rapports potentiels entre cet habitant habituel des plages et l'érotisme des situations :

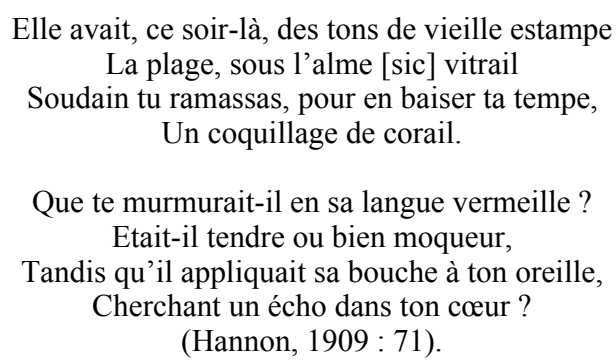

Il arrive à métamorphoser ses sentiments d'amoureux infatigable à travers des termes maritimes. Dans " Grains de beauté », bel exemple de poème érotique, ses « vœux » : « seraient de voir vers eux ${ }^{2}$ mon cœur enfler ses voiles », et son cœur « embarquerait d'ineffables plaisirs » (Hannon, 1909: 77).

Dans le dernier poème «P.P.C.», Hannon «prend congé », il laisse sa carte de visite pour dire adieu à cette Mer qu'il voit comme une « héroïne » :

\author{
Fin de saison !... Rentrent at home \\ Les villégiatureurs frileux \\ Qui s'enfuient, ô soleil fantôme, \\ Soufflant dans leurs pauvres doigts bleus... \\ Accompagnons-les, ma Musette \\ Quittons le flot, vraiment amer, \\ Après la suprême risette \\ A ton héroïne, la Mer \\ (Hannon, 1909 : 93).
}

Si la mer reste le lieu privilégié des amours de jeunesse de Rodenbach, elle ne sera qu'un paysage, un décor où Théodore Hannon comble sa hantise des amours éphémères, des amours charnels où rarement prennent place les idéalisations ou la rêverie. La femme est une figurine qui embellit la côte " élégante » de la mer du Nord et dont il profite poussé par ses affres charnelles. Au Clair de la dune, est un texte érotique qui, comme Maingueneau le définit: " est toujours pris dans la tentation de l'esthétisme, tenté de convertir la suggestion sexuelle en contemplation de pures formes » (Maingueneau, $2007: 18$ ).

Même si elles ont été considérées comme des œuvres mineures, La Mer élégante et Au clair de la dune ne doivent pas être délaissées car elles contiennent un univers riche en possibilités poétiques. Les deux recueils ne sont pas de simples documents

\footnotetext{
${ }^{2}$ Les yeux de l'aimée.
} 
artistiques de la vie de la bourgeoisie fin de siècle, Rodenbach et Hannon ont réussi à faire une belle interprétation de la mer à travers leur regard d'amoureux infatigables.

\section{RÉFÉRENCES BIBLIOGRAPHIQUES}

Berg, Ch., (1988) Lettres françaises de Belgique, t. II. Paris/Gembloux, Duculot.

Blain-Pinel, M., (2003) La Mer, miroir d'infini. Rennes, Presses Universitaires de Rennes.

Bodson-Thomas, A., (1942) L'Esthétique de Georges Rodenbach. Bruxelles, Académie Royale de Langue et Littérature Françaises de Belgique.

Delsemme, P., (1999) Nouvelle Bibliographie nationale. Bruxelles, Académie Royale des Sciences, des Lettres et des Beaux-Arts de Belgique.

Ensor, J., (1921) Les Écrits de James Ensor. Bruxelles, Éditions Sélection.

Gilsoul, R., (1936) La Théorie de l'art pour l'art chez les écrivains belges de 1880 à nos jours. Bruxelles, Académie Royale de Langue et de Littérature françaises de Belgique.

Gilkin, I., (2000) Mémoires inachevés. Bruxelles, Labor.

Hannon, Th., (1884) Rimes de joie. Bruxelles, Kistemaeckers.

Hannon, Th., (1909) Au clair de la dune. Bruxelles, Oscar Lamberty éd.

Le Guern, M., (1973) Sémantique de la métaphore et de la métonymie. Paris, Larousse.

Lemonnier, C., (1945) Une Vie d'écrivain. Bruxelles, Labor.

Maes, P., (1952) Georges Rodenbach 1855-1898. Bruxelles, Académie Royale de Langue et de Littérature françaises de Belgique.

Maingueneau, D., (2007) La Littérature pornographique. Paris, Armand Colin.

Rodenbach, G., (1881) La Mer élégante. Paris, Alphonse Lemerre éd.

Rodenbach, G., (1884) L'Hiver mondain. Bruxelles, Kistemaeckers. 